
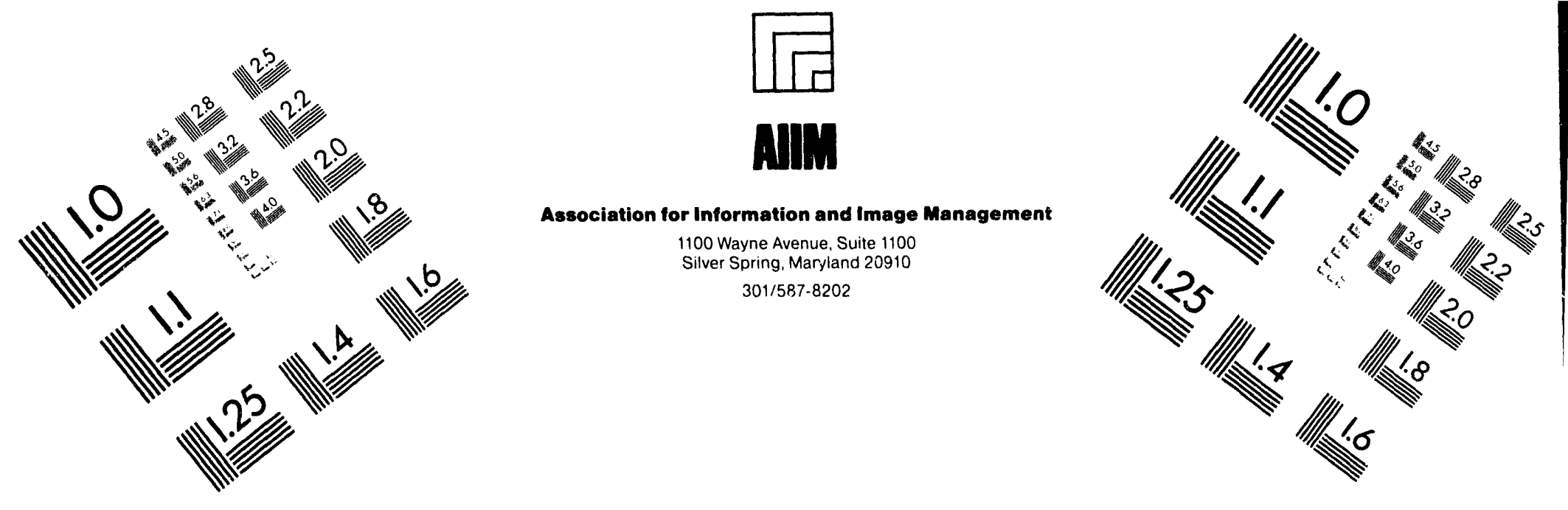

\title{
Centimeter
}

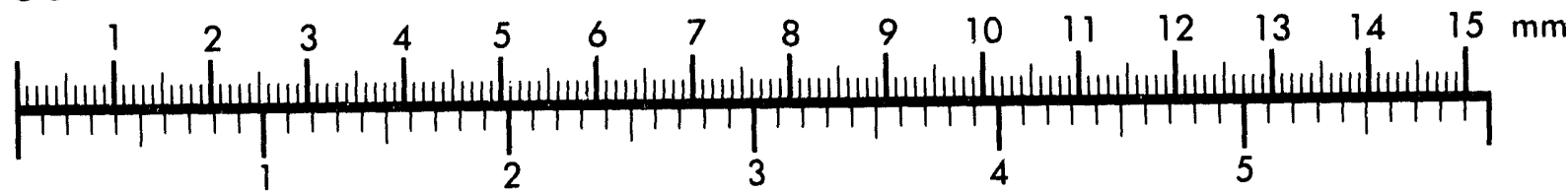
Inches
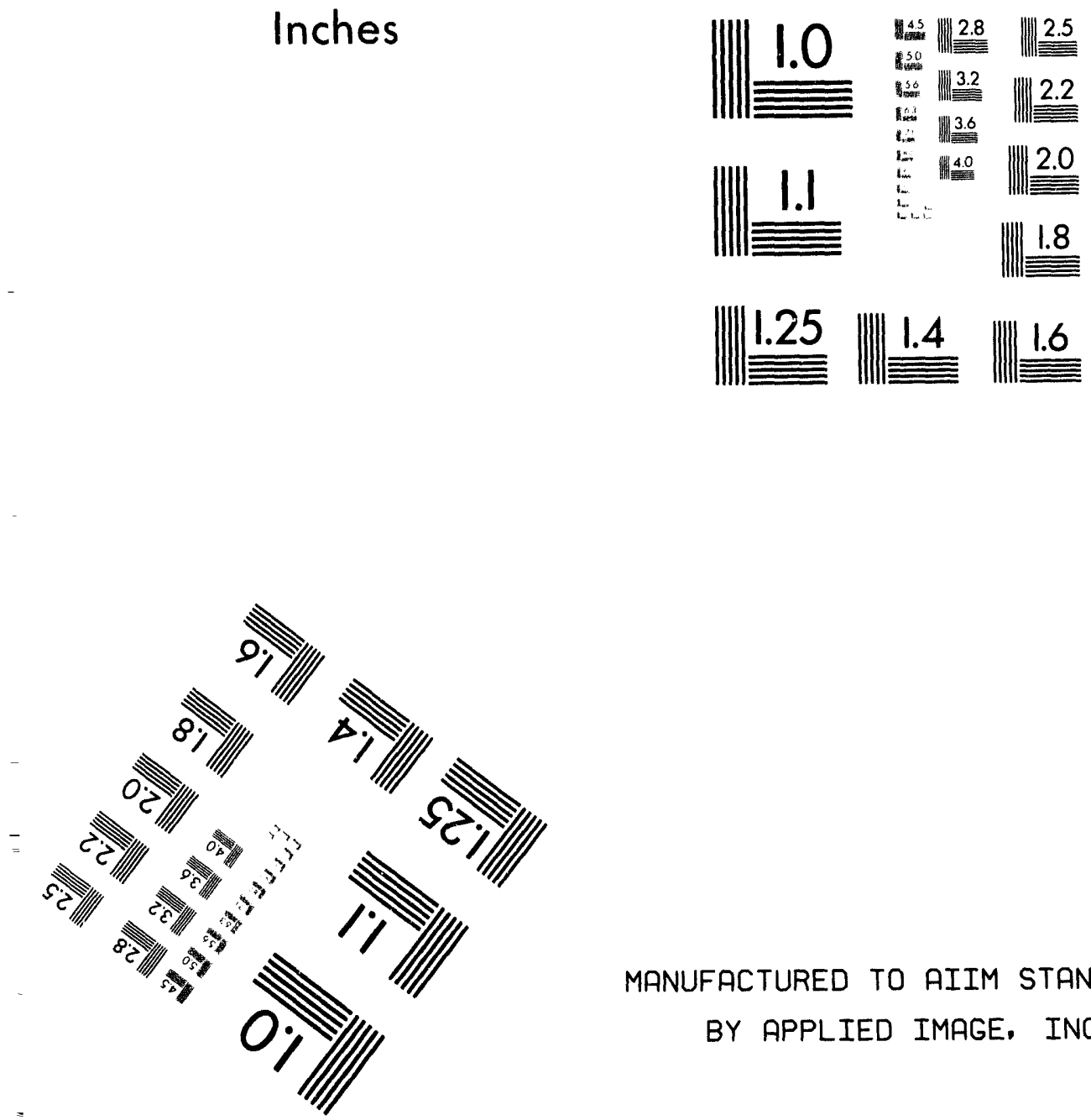

MANUFACTURED TO AIIM STANDARDS

BY APPLIED IMAGE. INC.

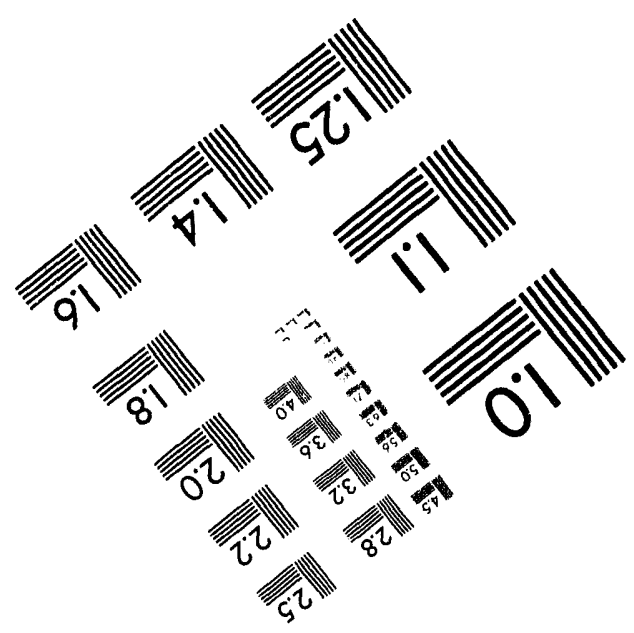



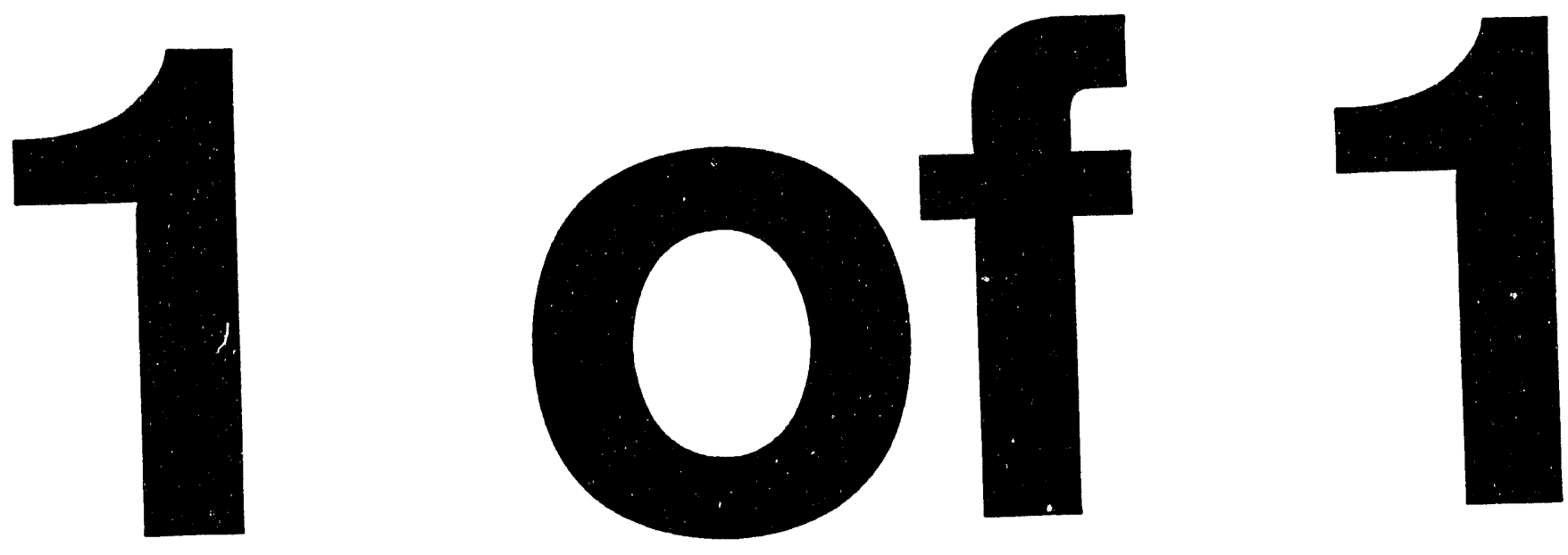


\title{
Conf $-940507-36$
}

UCRL-JC-117463

PREPRINT

\section{Pauli-Blocking Effects in Neutron-Alpha Reactions}

\author{
M. Avrigeanu \\ V. Avrigeanu \\ A.N. Antonov \\ M.B. Chadwick \\ P.E. Hodgson \\ M.V. Stoitsov
}

This paper was prepared for submittal to the International Conference on Nuclear Data for Science and Technology

Gatlinburg, TN

May 9-13, 1994

June 1, 1994

This is a preprint of a paper intended for publication in a joumal or proceedings. Since changes may be made before publication, this preprint is made available with the understanding that it will not be cited or reproduced without the permission of the author. 


\section{DISCLAIMER}

This document was prepared as an account of work sponsored by an agency of the United States Government. Neither the United States Government nor the University of California nor any of their employees, makes any warranty, express or implied, or assumes any legal liability or responsibility for the accu racy, completeness, or usefulness of any information, apparatus, product, or process disdosed, or represents that its use would not infringe privately owned rights. Reference herein to an y specific commercial products, process, or service by trade name, trademark, manufacturer, or otherwise, does not necessarily constitute or imply its endorsement, recommendation, or favoring by the United States Government or the University of California. The views and opinions of authors expressed herein do not necessarily state or rellect those of the United States Government or the University of California, and shall not be used for advertising or product endorsement purposes. 


\title{
PAULI-BLOCKING EFFECTS IN NEUTRON-ALPHA REACTIONS
}

\author{
M. Avrigeanu ${ }^{(1,4)}$, V. Avrigeanu(1) A.N. Antonov ${ }^{(2,4)}$, \\ M.B. Chadwick ${ }^{(3)}$, P.E. Hodgson ${ }^{(4)}$, and M.V. Stoitsov ${ }^{(2)}$ \\ (1) Institute of Physics and Nuclear Engineering, Bucharest 76900, Romania \\ (2) Institute of Nuclear Research and Nuclear Energy, Sofia 1784, Bulgaria \\ (3) University of California, Lawrence Livermore National Laboratory, Livermore, CA 94550, USA \\ (4) Nuclear Physics Laboratory, Department of Physics, University of Oxford, Oxford OX1 3RH, U.K.
}

\begin{abstract}
We present a knockout model for direct $(n, \alpha)$ reactions in which the residual nucleus is left in a continuum excited state. The interaction of the neutron with a preformed alpha particle inside the nucleus is related to the free neutron-alpha scattering cross-section, with modifications to account for nuclear medium effects, including Fermi motion, Pauli-blocking, and barrier penetration. Phase space restrictions for the four nucleons of the alphaparticle after the knockout are imposed by a Pauliblocking function. We apply this model, along with evaporation contributions, to analyze excitation functions of $(n, \alpha)$ reactions on ${ }^{48} \mathrm{Ti},{ }^{51} \mathrm{~V},{ }^{52} \mathrm{Cr}$, ${ }^{54} \mathrm{Fe},{ }^{55} \mathrm{Mn}$, and ${ }^{59} \mathrm{Co}$. Good agreement is obtained between our calculations and experimental measurements. Values for the local Fermi energy in the region from where knockout occurs indicate a surface reaction.
\end{abstract}

\section{The Model}

Nucleon-alpha reactions provide important infor mation on nuclear reaction mechanisms and on clustering in nuclei. There are a variety of competing mechanisms that contribute to the measured cross-sections: compound nucleus processes at low energies; and direct and preequilibrium processes at higher energies. At higher energies, reactions to the continuum are dominated by the knockout mechanism $[1,2]$, since the density of final states increases more rapidly than those corresponding to pick-up process.

Our model for $(n, \alpha)$ reactions has close similarities to quasi-free scattering models of nucleon and alpha particle emission $[3,4]$. The interaction of the incident nucleon with the (preformed) alpha particle is reiated to the free nucleon-alpha scattering cross-section. Modifications are then applied to account for nuclear medium effects, notably the Fermi momentum of the struck alpha particle, the Pauliblocking effects, and the influence of the residual nucleus nuclear and Coulomb barrier on the emitted alpha particle. Such an approach is also the basis of most intranuclear cascade models of nuclear reactions. In this work, however, we concentrate on the first-step "direct" part of the cascade, which is dominant for the energies we consider.

The influence of phase space restrictions in the nucleus can be expressed using a Pauli-blocking function, as in Chadwick et al.'s analysis of quasideuteron photoabsorption [5]. As in [5], it is supposed that the $(n, \alpha)$ cross-section is proportional to the available phase space, and Fermi-gas state densities are used. We require that the two protons and two neutrons of the preformed alphaparticle in the nucleus (with a preformation factor $\phi_{\alpha}$ ) after leaving the nucleus will all have energies greater than the Fermi-energy $\epsilon_{F}$. The direct component of the $\alpha$-particle emission cross-section can then be written as

$$
\sigma_{D C}=\phi_{\alpha} \sigma_{(n, \alpha)}^{\text {free }}\left(\epsilon_{\text {inc }}\right) f\left(\epsilon_{\text {inc }}\right),
$$

where $f\left(\epsilon_{\text {inc }}\right)$ is the Pauli-blocking function that depends on $\epsilon_{\text {inc }}$ (the energy of the incident neutron), $\sigma_{(n, \alpha)}^{\text {free }}$ is the free neutron-alpha cross-section, and $\phi_{\alpha}$ is the preformation factor. Using a Fermi-gas momentum distribution for the alpha-part les,

$$
f\left(\epsilon_{\mathrm{inc}}\right)=\int_{0}^{\iota_{F}^{\alpha}} \rho_{\alpha}\left(\epsilon_{\alpha}\right) F\left(\epsilon_{\alpha}+\epsilon_{\mathrm{inc}}\right) T\left(\epsilon_{\alpha}+\epsilon_{\mathrm{inc}}\right) d \epsilon_{\alpha},
$$

where $\epsilon_{\alpha}$ is the energy of the preformed alphaparticle, relative to the bottom of the nuclear well, $\rho_{\alpha}\left(\epsilon_{\alpha}\right)$ is the alpha-particle state density, $F\left(\epsilon_{\alpha}+\right.$ $\left.\epsilon_{\text {inc }}\right)$ is the Pauli-blocking factor, and $T\left(\epsilon_{\alpha}+\epsilon_{\text {inc }}\right)$ 


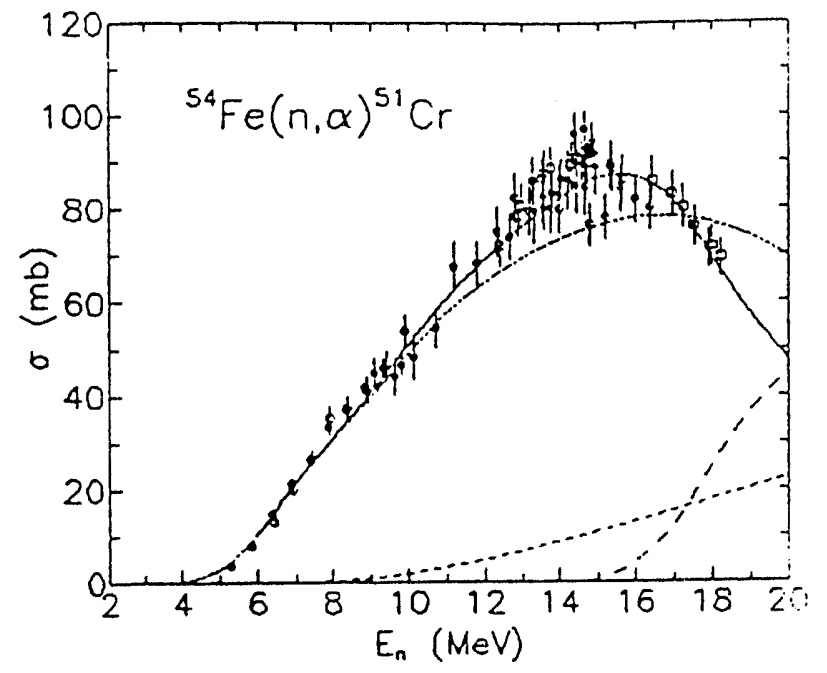

Figure 1: The excitation function of the ${ }^{54} \mathrm{Fe}(\mathrm{n}, \alpha){ }^{51} \mathrm{Cr}$ reaction. We show the calculated contributions: $\sigma_{D C}$, (dashed line); $\sigma_{H F}$ (dotted line); and $\sigma\left(n, \alpha n^{\prime}\right)$ (long-dashed line). The full calculation given by the solid line is $\sigma(n, \alpha)=$ $\sigma_{D C}+\sigma_{H F}-\sigma\left(n, \alpha n^{\prime}\right)$. References to the works from which the experimental data are taken are given in the text.

is the transmission coefficient for the excited alphaparticle to escape the nucleus (which we average for a range of angular momenta). We assume that the alpha-particle state density has a Fermi-gas model form, which is consistent with our use of a Pauliblocking factor given by

$$
F(E)=\frac{\rho^{P}(4 p, E)}{\rho(4 p, E)}
$$

defined as the ratio of the four-particle Fermi-gas state densities in which the Pauli blocking is included $\left(\rho^{P}(4 p, E)\right)$ and ignored $(\rho(4 p, E))[5]$.

\section{Results}

We apply our model to analyze $(n, \alpha)$ excitation functions measured using activation techniques. To the direct contributions calculated with the above model, we add a compound nucleus contribution from the Hauser-Feshbach theory $[6,7]$. If there is sufficient energy for subsequent particle decay following alpha emission, we isolate the $(n, \alpha)$ crosssection by subtracting such contributions. When calculating $\sigma_{D C}$ with our model, the free elastic

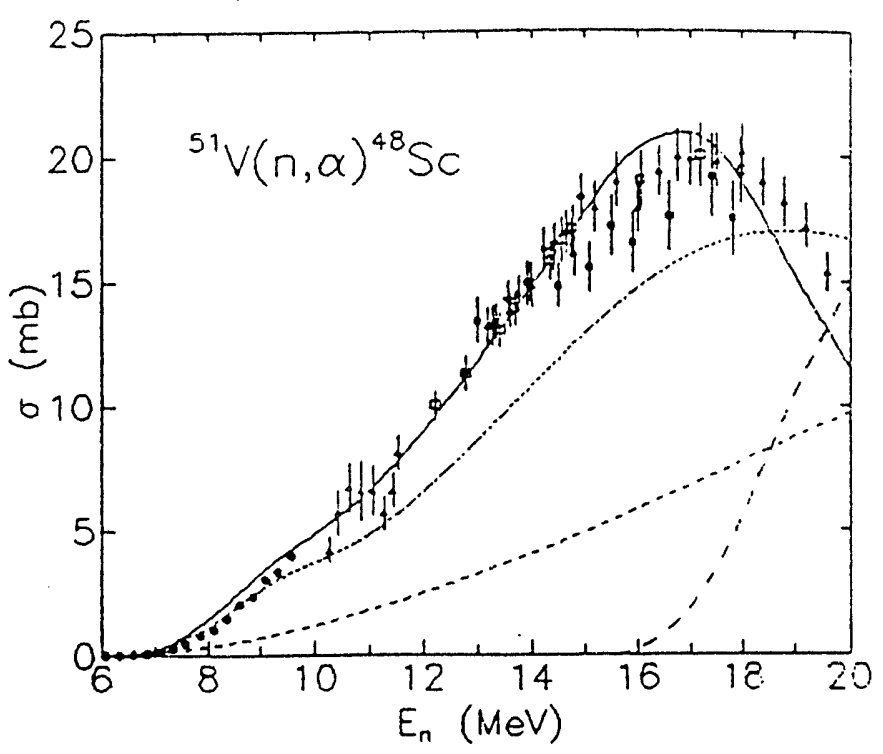

Figure 2: The same as in Fig. 1 for the ${ }^{51} \mathrm{~V}(n, \alpha)^{48} \mathrm{Sc}$ reaction.

Table 1: Values of $\epsilon_{F}$, the nucleon Fermi-energy (in $\mathrm{MeV}$ ), for a preformation factor $\phi_{\alpha}=0.30$. The low values of the Fermi energy indicate a nuclear surface reaction

\begin{tabular}{lllllll}
\hline & ${ }^{51} \mathrm{~V}$ & ${ }^{55} \mathrm{Mn}$ & ${ }^{59} \mathrm{Co}$ & ${ }^{48} \mathrm{Ti}$ & ${ }^{52} \mathrm{Cr}$ & ${ }^{54} \mathrm{Fe}$ \\
\hline$\epsilon_{F}$ & 9.0 & 7.0 & 6.0 & 4.0 & 8.0 & 4.5 \\
\hline
\end{tabular}

$(n, \alpha)$ cross-section was taken from Ref. [8], and the transmission coefficients from the optical potential in Ref. [9]. We have taken the value of the preformation factor as $\phi_{\alpha}=0.30$ in accordance with Refs. $[6,10,11]$.

The calculations of the $(n, \alpha)$ cross-section enable us to study the dependence of the results on the nucleon Fermi energy $\epsilon_{F}$. This parameter is obtained by fitting the calculated excitation function to the experimental data. We have analyzed excitation functions of $(n, \alpha)$ reactions on ${ }^{54} \mathrm{Fe},{ }^{51} \mathrm{~V},{ }^{55} \mathrm{Mn}$, ${ }^{59} \mathrm{Co},{ }^{48} \mathrm{Ti}$ and ${ }^{52} \mathrm{Cr}$. As an example of our calculations, we show in Fig. 1 the ${ }^{54} \mathrm{Fe}(n, \alpha)$ reaction, in Fig. 2 the ${ }^{51} \mathrm{~V}(n, \alpha)$ reaction, and in Fig. 3 the ${ }^{59} \mathrm{Co}$ $(n, \alpha)$ reaction. Experimental measurements from Refs. $[12,13]$ are compared with our model calculation. Our calculation of the $(n, \alpha)$ cross-section (solid line) is the sum of Hauser-Feshbach (dotted line) and our direct component (dashed line), subtracting secondary alphas which follow primary neutron emission (long-dashed line). The calculations are seen to describe the measurements well. 


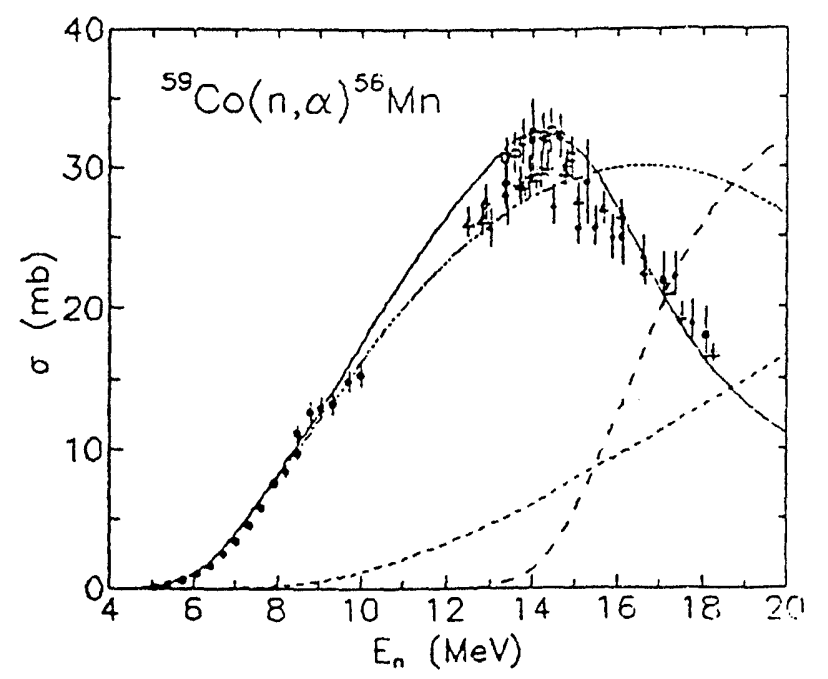

Figure 3: The same as in Fig.1 for the ${ }^{59} \mathrm{Co}(n, \alpha){ }^{56} \mathrm{Mn}$ reaction.

The optimum values of the nucleon Fermi are given in Table 1. According to the local density approximation the values of $\epsilon_{F}$ from 9.0 to 4.0 $\mathrm{MeV}$ correspond to nuclear surface densities from $\rho=0.106 \rho_{0}$ to $\rho=0.032 \rho_{0}$ (when $\epsilon_{F}=40 \mathrm{MeV}$ at $\left.\rho=\rho_{0}\right)$ and agree with the range $(4-8 \mathrm{MeV})$ found in the QFS-model [4] when applied to (nucleon, $\alpha$ ) reactions. The sensitivity of the cross-section to variations of $\phi_{\alpha}$ and $\epsilon_{F}$ was studied and it was found that the fit is essentially unaffected by correlated changes in these two parameters. Thus the parameter values $(0.20,7.0),(0.30,9.0)$, and $(0.40$, 10.5) for $\left(\phi_{\alpha}, \epsilon_{F}\right)$ give the same cross-section for the ${ }^{51} \mathrm{~V}(n, \alpha)$-reaction. For a particular value of $\phi_{\alpha}$, we estimate the uncertainty in $\epsilon_{F}$ to be about $0.5-1.0 \mathrm{MeV}$.

In summary, we have shown that our model accurately describes the direct components of the $(n, \alpha)$ cross-section (and the total excitation function after including statistical components). The values we obtain for the local Fermi-energy are consistent with results from other investigations, and indicate that alpha knockout occurs from the surface region. As a next step in this work, we plan to investigate the use of this model to calculate emission spectra of alpha particles.

This work was funded in part by the ECC (Contract No. ERB-CIPA-CT-92-2159), by the Bulgarian National Science Foundation (Contract No. Ph.-32) (A.N.A.), under the auspices of the U.S.
Department of Energy by the Lawrence Livermore National Laboratory under Contract No. W'-7405ENG-48 (M.B.C.), by the Royal Society (M.A.), and by the agreement of the Royal Society with the Bulgarian Academy of Sciences (A.N.A.).

\section{References}

[1] E. Gadioli and P.E. Hodgson, Rep. Prog. Phys. 52, 247 (1989); E. Gadioli and P.E. Hodgson, Pre-Equilibrium Nuclear Reactions, Clarendon Press, Oxford (1992).

[2] A. Ferrero, E. Gadioli, E. Gadioli Erba, I. Iori, N. Mohlo, L. Zetta, Z. Phys. A293, 123 (1979).

[3] A. Mignerey, M. Blann, W. Scobel, Nucl. Phys. A273, 125 (1976).

[4] W. Scobel, M. Blann, A. Mignerey, Nucl. Phys. A287, 301 (1977).

[5] M.B. Chadwick, P. Oblozinsky, P.E. Hodgson, G. Reffo, Phys. Rev. C44, 814 (1991).

[6] M. Avrigeanu, M. Ivascu and V. Avrigeanu, Z. Phys. A235, 299 (1990).

[7] M. Avrigeanu, V. Avrigeanu and P. E. Hodgson, in Proc. of Int. Adv. Courses on Frontier Topics in Nuclear Physics, Predeal, Romania, September 1993, Eds. W. Scheid, A. Sandulescu, Plenum, N.Y. (in press).

[8] JAERI-M 90-099 (JENDL-3); K. Shibata, J. of Nucl. Sci. and Technology 27[1], 81 (1990).

[9] V. Avrigeanu, P. E. Hodgson and $M$. Avrigeanu, Phys. Rev. C49, (1994) (in print).

[10] S.M. Qaim, M. Uhl, N.I. Molla, H. Liskien, Phys. Rev. C46, 1398 (1992).

[11] N. I. Molli, S. M. Qaim, M. Uhl, Phys. Rev. C42, 1540 (1990)

[12] V. Avrigeanu, "Calculations of Excitation Functions of $(n, \alpha)$ Reactions", IAEA Research Co-ordination Meeting on "Improvement of Measurements, Theoretical Computations and Evaluations of Neutron Induced Helium Production Cross Sections", Debrecen, Hungary, 17-19 November 1992 (and references therein).

[13] V. Mclane, C.L. Dunford, P.E. Rose, Neutron Cross Sections, vol.2, Neutron Cross Section Curves, Academic Press, Inc., Boston, (1988). 

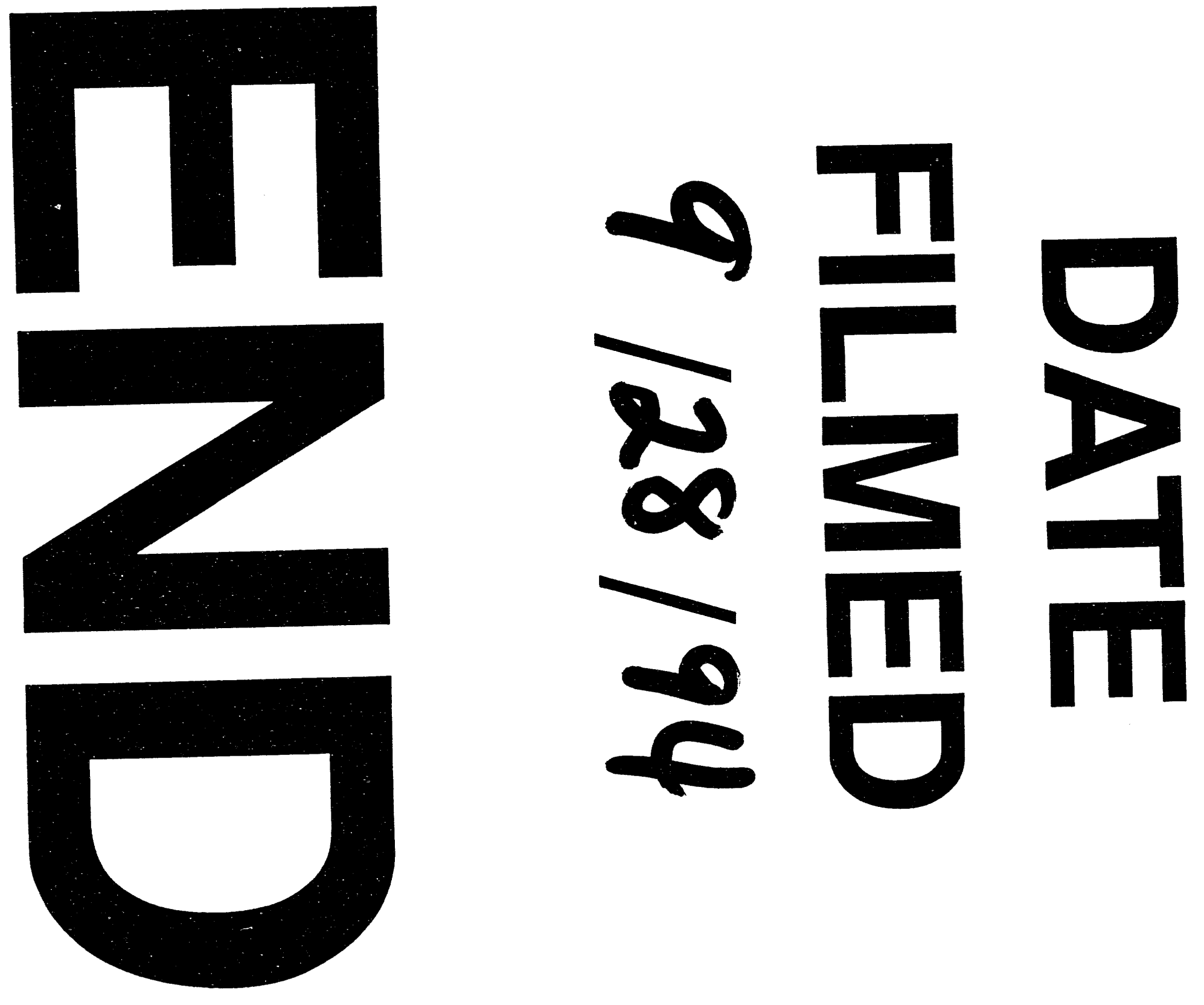
